Article

\title{
On the Asymptotic Behavior of D-Solutions to the Displacement Problem of Linear Elastostatics in Exterior Domains
}

\author{
Vincenzo Coscia \\ Department of Mathematics and Computer Science, University of Ferrara, Via Machiavelli 30, 44121 Ferrara, \\ Italy; vincenzo.coscia@unife.it
}

Received: 15 November 2019; Accepted: 30 December 2019; Published: 3 January 2020

check for updates

\begin{abstract}
We study the asymptotic behavior of solutions with finite energy to the displacement problem of linear elastostatics in a three-dimensional exterior Lipschitz domain.
\end{abstract}

Keywords: non-homogeneous elasticity; exterior domains; existence and uniqueness theorems; asymptotic behavior; Stokes' paradox

MSC: Primary 74B05; 35J47; 35J57; Secondary 35J20

\section{Introduction}

The displacement problem of elastostatics in an exterior Lipschitz domain $\Omega$ of $\mathbb{R}^{3}$ consists of finding a solution to the equations [1] (Notation-Unless otherwise specified, we will use the notation of the classical monograph [1] by M.E. Gurtin. In particular, $(\operatorname{div} \mathbf{C}[\nabla \boldsymbol{u}])_{i}=\partial_{j}\left(\mathrm{C}_{i j h k} \partial_{k} u_{h}\right)$, Lin is the space of second-order tensors (linear maps from $\mathbb{R}^{3}$ into itself) and Sym, Skw are the spaces of the symmetric and skew elements of Lin respectively; if $E \in$ Lin and $v \in \mathbb{R}^{3}$, $E v$ is the vector with components $E_{i j} v_{j} . B_{R}=\left\{x \in \mathbb{R}^{3}: r=|x|<R\right\}, T_{R}=B_{2 R} \backslash B_{R}, \complement B_{R}=\mathbb{R}^{3} \backslash \overline{B_{R}}$ and $B_{R_{0}}$ is a fixed ball containing $\partial \Omega$. If $f(x)$ and $\phi(r)$ are functions defined in a neighborhood of infinity, then $f(x)=o(\phi(r))$ means that $\lim _{r \rightarrow+\infty}(f / \phi)=0$. To lighten up the notation, we do not distinguish between scalar, vector, and second-order tensor space functions; $c$ will denote a positive constant whose numerical value is not essential to our purposes.)

$$
\begin{array}{rlrl}
\operatorname{div} \mathbf{C}[\nabla \boldsymbol{u}] & =\mathbf{0} & & \text { in } \Omega, \\
\boldsymbol{u} & =\hat{\boldsymbol{u}} & & \text { on } \partial \Omega, \\
\lim _{R \rightarrow+\infty} \int_{\partial B} \boldsymbol{u}(R, \sigma) d \sigma & =\mathbf{0}, &
\end{array}
$$

where $\boldsymbol{u}$ is the (unknown) displacement field, $\hat{\boldsymbol{u}}$ is an (assigned) boundary displacement, $B$ is the unit ball, $\mathbf{C} \equiv\left[\mathrm{C}_{i j h k}\right]$ is the (assigned) elasticity tensor, i.e., a map from $\Omega \times \operatorname{Lin} \rightarrow$ Sym, linear on Sym and vanishing in $\Omega \times$ Skw. We shall assume $\mathbf{C}$ to be symmetric, i.e.,

$$
\boldsymbol{E} \cdot \mathbf{C}[\boldsymbol{L}]=\boldsymbol{L} \cdot \mathbf{C}[\boldsymbol{E}] \quad \forall \boldsymbol{E}, \boldsymbol{L} \in \mathrm{Lin},
$$

and positive definite, i.e., there exists positive scalars $\mu_{0}$ and $\mu_{e}$ (minimum and maximum elastic moduli [1]) such that

$$
\mu_{0}|\boldsymbol{E}|^{2} \leq \boldsymbol{E} \cdot \mathbf{C}[\boldsymbol{E}] \leq \mu_{e}|\boldsymbol{E}|^{2}, \quad \forall \boldsymbol{E} \in \text { Sym, a.e. in } \Omega \text {. }
$$


Let $D^{1, q}(\Omega), D_{0}^{1, q}(\Omega)(q \in[1,+\infty))$ be the completion of $C_{0}^{\infty}(\bar{\Omega})$ and $C_{0}^{\infty}(\Omega)$, respectively, with respect to the norm $\|\nabla \boldsymbol{u}\|_{L^{q}(\Omega)}$.

We consider solutions $u$ to equations (1) with finite Dirichlet integral (or with finite energy) that we call $D$-solutions analogous with the terminology used in viscous fluid dynamics (see [2]). More precisely, we say that $u \in D^{1,2}(\Omega)$ is a $D$-solution to equation (1)

$$
\int_{\Omega} \nabla \boldsymbol{\varphi} \cdot \mathbf{C}[\nabla \boldsymbol{u}]=0, \quad \forall \boldsymbol{\varphi} \in D_{0}^{1,2}(\Omega) .
$$

A $D$-solution to system (1) is a $D$-solution to equation $(1)_{1}$, which satisfies the boundary condition in the sense of trace in Sobolev's spaces and tends to zero at infinity in a mean square sense [2]

$$
\lim _{R \rightarrow+\infty} \int_{\partial B}|\boldsymbol{u}(R, \sigma)|^{2} d \sigma=0 .
$$

If $\boldsymbol{u}$ is a $D$-solution to $(1)_{1}$, then the traction field on the boundary is

$$
s(\boldsymbol{u})=\mathbf{C}[\nabla \boldsymbol{u}] \boldsymbol{n}
$$

where a well defined field of $W^{-1 / 2,2}(\partial \Omega)$ exists and the following generalized work and energy relation [1] holds

$$
\int_{\Omega \cap B_{R}} \nabla \boldsymbol{u} \cdot \mathbf{C}[\nabla \boldsymbol{u}]=\int_{\partial \Omega} \boldsymbol{u} \cdot \boldsymbol{s}(\boldsymbol{u}),
$$

where abuse of notation $\int_{\partial \Omega} \boldsymbol{u} \cdot \boldsymbol{s}(\boldsymbol{u})$ means the value of the functional $\boldsymbol{s}(\boldsymbol{u}) \in W^{-1 / 2,2}(\partial \Omega)$ at $\boldsymbol{u} \in$ $W^{1 / 2,2}(\partial \Omega)$, and $n$ is the unit outward (with respect to $\Omega$ ) normal to $\partial \Omega$.

If $\hat{\boldsymbol{u}} \in W^{1 / 2,2}(\partial \Omega)$, denoting by $\boldsymbol{u}_{0} \in D^{1,2}(\Omega)$ an extension of $\hat{\boldsymbol{u}}$ in $\Omega$ vanishing outside a ball, then (1) $)_{1,2}$ is equivalent to finding a field $\boldsymbol{u} \in D_{0}^{1,2}(\Omega)$ such that

$$
\int_{\Omega} \nabla \boldsymbol{\varphi} \cdot \mathbf{C}[\nabla \boldsymbol{v}]=-\int_{\Omega} \nabla \boldsymbol{\varphi} \cdot \mathbf{C}\left[\nabla \boldsymbol{u}_{0}\right], \quad \forall \boldsymbol{\varphi} \in D_{0}^{1,2}(\Omega) .
$$

Since the right-hand side of (6) defines a linear and continuous functional on $D_{0}^{1,2}(\Omega)$, and by the first Korn inequality (see [1] Section 13)

$$
\int_{\Omega}|\nabla v|^{2} \leq \frac{2}{\mu_{0}} \int_{\Omega} \nabla \boldsymbol{v} \cdot \mathbf{C}[\nabla \boldsymbol{v}]
$$

by the Lax-Milgram lemma, (6) has a unique solution $\boldsymbol{v}$, and the field $\boldsymbol{u}=\boldsymbol{v}+\boldsymbol{u}_{0}$ is a $D$-solution to $(1)_{1,2}$. It satisfies $(1)_{3}$ in the following sense (see Lemma 1)

$$
\int_{\partial B}|\boldsymbol{u}(R, \sigma)|^{2} d \sigma=o\left(R^{-1}\right) .
$$

Moreover, $u$ exhibits more regularity properties provided $C, \partial \Omega$ and $\hat{u}$ are more regular. In particular, if $\mathbf{C}, \hat{\boldsymbol{u}}$ and $\partial \Omega$ are of class $C^{\infty}$, then $\boldsymbol{u} \in C^{\infty}(\bar{\Omega})$ [3].

If $\mathbf{C}$ is constant, then existence and regularity hold under the weak assumption of strong ellipticity [1], i.e.,

$$
\mu_{0}|\boldsymbol{a}|^{2}|\boldsymbol{b}|^{2} \leq a \cdot \mathbf{C}[\boldsymbol{a} \otimes \boldsymbol{b}] \boldsymbol{b}, \quad \forall \boldsymbol{a}, \boldsymbol{b} \in \mathbb{R}^{3} .
$$

As far as we are aware, except for the property (7), little is known about the convergence at infinity of a $D$-solution and, in particular, whether or under what additional conditions (7) can be improved. 
The main purpose of this paper is just to determine reasonable conditions on $\mathbf{C}$ assuring that (7) can be improved.

We say that $\mathbf{C}$ is regular at infinity if there is a constant elasticity tensor $\mathbf{C}_{0}$ such that

$$
\lim _{|x| \rightarrow+\infty} \mathbf{C}(x)=\mathbf{C}_{0}
$$

Let $\mathfrak{C}_{0}$ and $\mathfrak{C}$ denote the linear spaces of the $D$-solutions to the equations

$$
\begin{array}{rlrl}
\operatorname{div} \mathbf{C}[\nabla \boldsymbol{h}] & =\mathbf{0} & & \text { in } \Omega, \\
\boldsymbol{h} & =\boldsymbol{\tau} & & \text { on } \partial \Omega, \\
\lim _{R \rightarrow+\infty} \int_{\partial B}|\boldsymbol{h}(R, \sigma)|^{2} d \sigma & =\mathbf{0}, &
\end{array}
$$

for all $\tau \in \mathbb{R}^{3}$ and

$$
\begin{array}{rlrl}
\operatorname{div} \mathbf{C}[\nabla \boldsymbol{h}] & =\mathbf{0} & & \text { in } \Omega, \\
\boldsymbol{h} & =\boldsymbol{\tau}+\boldsymbol{A x} & & \text { on } \partial \Omega, \\
\lim _{R \rightarrow+\infty} \int_{\partial B}|\boldsymbol{h}(R, \sigma)|^{2} d \sigma & =\mathbf{0}, &
\end{array}
$$

for all $\tau \in \mathbb{R}^{3}, A \in$ Lin, respectively.

The following theorem holds.

Theorem 1. Let $\boldsymbol{u}$ be the D-solution to (1). There is $q<2$ depending only on $\boldsymbol{C}$ such that

$$
\int_{\partial B}|\boldsymbol{u}(R, \sigma)|^{q} d \sigma=o\left(R^{q-3}\right) .
$$

If $\boldsymbol{C}$ is regular at infinity, then

$$
\int_{\partial B}|\boldsymbol{u}(R, \sigma)|^{q} d \sigma=o\left(R^{q-3}\right), \quad \forall q \in(3 / 2,+\infty),
$$

and

$$
\int_{\partial B}|\boldsymbol{u}(R, \sigma)|^{q} d \sigma=o\left(R^{q-3}\right), \forall q \in(1,2] \Longleftrightarrow \int_{\partial \Omega} \hat{\boldsymbol{u}} \cdot \boldsymbol{s}(\boldsymbol{h})=\mathbf{0}, \quad \forall \boldsymbol{h} \in \mathfrak{C}_{0} .
$$

Moreover, if

$$
\int_{\partial \Omega} \boldsymbol{C}[\hat{\boldsymbol{u}} \otimes \boldsymbol{n}]=\mathbf{0}, \quad \int_{\partial \Omega} \hat{\boldsymbol{u}} \cdot \boldsymbol{s}(\boldsymbol{h})=\mathbf{0}, \quad \forall \boldsymbol{h} \in \mathfrak{C},
$$

then

$$
\int_{\partial B}|\boldsymbol{u}(R, \sigma)| d \sigma=o\left(R^{-2}\right)
$$

\section{Preliminary Results}

In this section, we collect the main tools we need to prove Theorem 1.

Lemma 1. If $u \in D^{1, q}(\Omega)$, for $q \in[1,2]$, then

$$
\int_{\partial B}|\boldsymbol{u}(R, \sigma)|^{q} d \sigma \leq c(q) R^{q-3} \int_{C B_{R}}|\nabla \boldsymbol{u}|^{q}
$$


Moreover, if $q=2$, then, for $R \gg R_{0}$,

$$
\int_{C B_{R}} \frac{|\boldsymbol{u}|^{2}}{r^{2}} \leq 4 \int_{C B_{R}}|\nabla \boldsymbol{u}|^{2} .
$$

Proof. Lemma 1 is well-known (see, e.g., [2,4] and [5] Chapter II). We propose a simple proof for the sake of completeness. Since $D^{1,2}(\Omega)$ is the completion of $C_{0}^{\infty}(\bar{\Omega})$ with respect to the norm $\|\nabla \boldsymbol{u}\|_{L^{2}(\Omega)}$, it is sufficient to prove (17) and (18) for a regular field $u$ vanishing outside a ball. By basic calculus and Hölder inequality,

$$
\begin{aligned}
\int_{\partial B}|\boldsymbol{u}(R, \sigma)|^{q} d \sigma & =\int_{\partial B}\left|\int_{R}^{+\infty} \partial_{r} \boldsymbol{u}(r, \sigma) d r\right|^{q} d \sigma=\iint_{\partial B}\left|\int_{R}^{+\infty} r^{2 / q_{r}-2 / q} \partial_{r} \boldsymbol{u}(r, \sigma) d r\right|^{q} d \sigma \\
& \leq\left\{\int_{\partial B} d \sigma \int_{R}^{+\infty}|\nabla \boldsymbol{u}(r, \sigma)|^{q} r^{2} d r\right\}\left\{\int_{\partial B}\left|\int_{R}^{+\infty} r^{-\frac{2}{q-1}} d r\right|^{q-1} d \sigma\right\} .
\end{aligned}
$$

Hence, (17) follows by a simple integration.

From

$$
\int_{\complement B_{R}} \frac{|\boldsymbol{u}|^{2}}{r^{2}}=\int_{R}^{+\infty} \partial_{r}\left(r \int_{\partial B}|\boldsymbol{u}(r, \sigma)|^{2} d \sigma\right)-2 \int_{\complement B_{R}} \frac{\boldsymbol{u}}{r} \cdot \partial_{r} \boldsymbol{u}
$$

by Schwarz's inequality, one gets

$$
\int_{\complement B_{R}} \frac{|\boldsymbol{u}|^{2}}{r^{2}} \leq 2\left\{\int_{C B_{R}} \frac{|\boldsymbol{u}|^{2}}{r^{2}} \int_{C B_{R}}|\nabla \boldsymbol{u}|^{2}\right\}^{1 / 2} .
$$

Hence, (18) follows at once.

Let $\mathbf{C}_{0}$ be a constant and strongly elliptic elasticity tensor. The equation

$$
\operatorname{div} \mathbf{C}_{0}[\nabla \boldsymbol{u}]=\mathbf{0}
$$

admits a fundamental solution $\mathfrak{U}(x-y)$ [6] that enjoys the same qualitative properties as the well-known ones of homogeneous and isotropic elastostatics, defined by

$$
u_{i j}(x-y)=\frac{1}{8 \pi \mu(1-v)|x-y|}\left[(3-4 v) \delta_{i j}+\frac{\left(x_{i}-y_{i}\right)\left(x_{j}-z_{j}\right)}{|x-y|^{2}}\right],
$$

where $\mu$ is the shear modulus and $v$ the Poisson ratio (see [1] Section 51). In particular, $\mathfrak{u}(x)=O\left(r^{-1}\right)$ and for $f$ with compact support (say) the volume potential

$$
\mathcal{v}[\boldsymbol{f}](x)=\int_{\mathbb{R}^{3}} \mathfrak{u}(x-y) \boldsymbol{f}(y) d v_{y}
$$

is a solution (in a sense depending on the regularity of $f$ ) to the system

$$
\operatorname{div} \mathrm{C}_{0}[\nabla \boldsymbol{u}]+f=\mathbf{0} \quad \text { in } \mathbb{R}^{3} .
$$

Let $\mathcal{H}^{1}$ denote the Hardy space on $\mathbb{R}^{3}$ (see [7] Chapter III). The following result is classical (see, e.g., [7]).

Lemma 2. $\nabla_{2} \mathcal{V}$ maps boundedly $L^{q}$ into itself for $q \in(1,+\infty)$ and $\mathcal{H}^{1}$ into itself. 
Lemma 3. Let $u$ be the D-solution to (1), Then, for $R \gg R_{0}$,

$$
\int_{\partial \Omega} \boldsymbol{s}(\boldsymbol{u})=\int_{\partial B_{R}} \boldsymbol{s}(\boldsymbol{u})
$$

and

$$
\int_{\partial \Omega} \boldsymbol{h} \cdot \boldsymbol{s}(\boldsymbol{u})=\int_{\partial \Omega} \hat{\boldsymbol{u}} \cdot \boldsymbol{s}(\boldsymbol{h}), \quad \forall \boldsymbol{h} \in \mathfrak{C},
$$

where $\mathfrak{C}$ is the space of $D$-solutions to system (11).

Proof. Let

$$
g(x)= \begin{cases}0, & |x|>2 R \\ 1, & |x|<R \\ R^{-1}(R-|x|), & R \leq|x| \leq 2 R\end{cases}
$$

with $R \gg R_{0}$. Scalar multiplication of both sides of $(1)_{1}$ by $g h,(2)$ and an integration by parts yield

$$
\int_{\partial \Omega} \boldsymbol{h} \cdot \boldsymbol{s}(\boldsymbol{u})-\int_{\partial \Omega} \hat{\boldsymbol{u}} \cdot \boldsymbol{s}(\boldsymbol{h})=\frac{1}{R} \int_{T_{R}}\left(\boldsymbol{u} \cdot \mathbf{C}[\nabla \boldsymbol{h}] \boldsymbol{e}_{r}-\boldsymbol{h} \cdot \mathbf{C}[\nabla \boldsymbol{u}] \boldsymbol{e}_{r}\right),
$$

where $e_{r}=x / r$. Since $R \leq|x| \leq 2 R$, by Schwarz's inequality,

$$
\begin{aligned}
& \left|\frac{1}{R} \int_{T_{R}} \boldsymbol{h} \cdot \mathbf{C}[\nabla \boldsymbol{u}] \boldsymbol{e}_{r}\right| \leq 2 \int_{T_{R}} r^{-1}\left|\boldsymbol{h} \cdot \mathbf{C}[\nabla \boldsymbol{u}] \boldsymbol{e}_{r}\right| \leq c\left\|r^{-1} \boldsymbol{h}\right\|_{L^{2}\left(T_{R}\right)}\|\nabla \boldsymbol{u}\|_{L^{2}\left(T_{R}\right)}, \\
& \left|\frac{1}{R} \int_{T_{R}} \boldsymbol{u} \cdot \mathbf{C}[\nabla \boldsymbol{h}] \boldsymbol{e}_{r}\right| \leq 2 \int_{T_{R}} r^{-1}\left|\boldsymbol{u} \cdot \mathbf{C}[\nabla \boldsymbol{h}] \boldsymbol{e}_{r}\right| \leq c\left\|r^{-1} \boldsymbol{u}\right\|_{L^{2}\left(T_{R}\right)}\|\nabla \boldsymbol{h}\|_{L^{2}\left(T_{R}\right)} .
\end{aligned}
$$

Hence, letting $R \rightarrow+\infty$ and taking into account Lemma 1, (23) follows.

Lemma 4. Let $\boldsymbol{u}$ be the D-solution to (1); then, for $R \gg R_{0}$,

$$
\int_{\partial \Omega}(\boldsymbol{x} \otimes \boldsymbol{s}(\boldsymbol{u})-\boldsymbol{C}[\hat{\boldsymbol{u}} \otimes \boldsymbol{n}])=\int_{\partial B_{R}}\left(\boldsymbol{x} \otimes \boldsymbol{s}(\boldsymbol{u})-\boldsymbol{C}\left[\boldsymbol{u} \otimes \boldsymbol{e}_{R}\right]\right),
$$

where $\mathfrak{C}$ is the space of D-solutions to system (11).

Proof. (25) is easily obtained by integrating the identity

$$
\mathbf{0}=\boldsymbol{x} \otimes \operatorname{div} \mathbf{C}[\nabla \boldsymbol{u}]=\operatorname{div}(\boldsymbol{x} \otimes \mathbf{C}[\nabla \boldsymbol{u}])-\mathbf{C}[\nabla \boldsymbol{u}]
$$

over $B_{R}$ and using the divergence theorem.

\section{Proof of Theorem 1}

Let $\vartheta(r)$ be a regular function, vanishing in $B_{R}$ and equal to 1 outside $B_{2 R}$, for $R \gg R_{0}$. The field $v=\vartheta u$ is a $D$-solution to the equation

$$
\operatorname{div} \mathbf{C}[\nabla \boldsymbol{v}]+f=\mathbf{0} \quad \text { in } \mathbb{R}^{3}
$$

with

$$
\boldsymbol{f}=-\mathbf{C}[\nabla \boldsymbol{u}] \nabla \vartheta-\operatorname{div} \mathbf{C}[\boldsymbol{u} \otimes \nabla \vartheta]
$$


Of course, $f \in L^{2}\left(\mathbb{R}^{3}\right)$ vanishes outside $T_{R}$. Let $\mathbf{C}_{0}$ be a strongly elliptic elasticity tensor. Clearly, $v$ is a $D$-solution to the system

$$
\operatorname{div} \mathbf{C}_{0}[\nabla \boldsymbol{v}]+\operatorname{div}\left(\mathbf{C}-\mathbf{C}_{0}\right)[\nabla \boldsymbol{v}]+f=\mathbf{0} \quad \text { in } \mathbb{R}^{3},
$$

which coincides with $u$ outside $B_{2 R}$. Since

$$
\nabla_{k} \mathcal{v}[f](x)=O\left(r^{-1-k}\right), \quad k \in \mathbb{N}, \quad \nabla_{k}=\underbrace{\nabla \ldots \nabla}_{k-\text { times }},
$$

by Lemma 2, the map

$$
\boldsymbol{w}^{\prime}(x)=\nabla \mathcal{V}\left[\left(\mathbf{C}-\mathbf{C}_{0}\right)[\nabla \boldsymbol{w}]\right](x)+\mathcal{V}[f](x)
$$

is continuous from $D^{1, q}$ into itself, for $q \in(3 / 2,+\infty)$. Choose

$$
\mathbf{C}_{0 i j h k}=\mu_{e} \delta_{i h} \delta_{j k}
$$

Since

$$
\left\|\nabla \mathcal{V}\left[\left(\mathbf{C}-\mathbf{C}_{0}\right)[\nabla \boldsymbol{w}]\right]\right\|_{D^{1, q}} \leq c(q) \frac{\mu_{e}-\mu_{0}}{\mu_{e}}\|\boldsymbol{w}\|_{D^{1, q}}
$$

and [7]

$$
\lim _{q \rightarrow 2} c(q)=1,
$$

the map (30) is contractive in a neighborhood of 2 and its fixed point must coincide with $v$. Hence, there is $q \in(1,2)$ such that $\boldsymbol{u} \in D^{1, q}(\Omega)$ and (12) is proved.

If $\mathbf{C}$ is regular at infinity, then by Lemma 1 and the property of $\vartheta$,

$$
\left\|\boldsymbol{v}^{\prime}-\boldsymbol{z}^{\prime}\right\|_{D^{1, q}} \leq c(q)\left\|\mathbf{C}-\mathbf{C}_{0}\right\|_{L^{\infty}\left(C S_{R_{0}}\right.}\|\boldsymbol{v}-\boldsymbol{z}\|_{D^{1, q}}
$$

Since the constant $c(q)$ is uniformly bounded in every interval $[a, b]$ and $\left\|\mathbf{C}-\mathbf{C}_{0}\right\|_{L^{\infty}\left(C S_{R_{0}}\right)}$ is sufficiently small, $u \in D^{1, q}$ for $\boldsymbol{q} \in(3 / 2,+\infty)$.

Assume that

$$
\int_{\partial \Omega} \hat{\boldsymbol{u}} \cdot s(\boldsymbol{h})=\mathbf{0}, \quad \forall \boldsymbol{h} \in \mathfrak{C}_{0} .
$$

By Lemma 3 , for $R \gg R_{0}$,

$$
\int_{\partial B_{R}} \boldsymbol{s}(\boldsymbol{u})=\int_{\partial B_{R}} \mathbf{C}[\nabla \boldsymbol{u}] \boldsymbol{e}_{R}=\mathbf{0} .
$$

Therefore, taking into account (27),

$$
\int_{\mathbb{R}^{3}} f=\int_{T_{R}} f=\int_{R}^{2 R} \vartheta^{\prime}(r) d r \int_{\partial B_{r}} \mathbf{C}[\nabla \boldsymbol{u}] \boldsymbol{e}_{r}=\mathbf{0},
$$

Since

$$
\mathcal{V}[f](x)=\int_{\mathbb{R}^{3}}[\mathfrak{u}(x-y)-\mathfrak{u}(y)] f(y) d v_{y}+\mathfrak{u}(x) \int_{\mathbb{R}^{3}} f
$$

by (33), Lagrange's theorem and (29)

$$
\nabla \mathcal{V}[f](x)=O\left(r^{-3}\right),
$$

so that

$$
\nabla \mathcal{V}[f] \in L^{q}, \quad q \in(1,2] .
$$


Then, by (31), the map (30) is contractive for $q$ in a right neighborhood of 1 so that

$$
\boldsymbol{u} \in D^{1, q}(\Omega), \quad q \in(1,2] .
$$

Conversely, if (35) holds, then a simple computation yields

$$
\int_{\partial \Omega} \boldsymbol{s}(\boldsymbol{u})=\int_{\mathbb{R}^{3}} \mathbf{C}[\nabla \boldsymbol{u}] \nabla g=-\frac{1}{R} \int_{T_{R}} \mathbf{C}[\nabla \boldsymbol{u}] \boldsymbol{e}_{r},
$$

where $g$ is the function (24). By Hölder's inequality,

$$
\frac{1}{R}\left|\int_{T_{R}} \mathbf{C}[\nabla \boldsymbol{u}] e_{r}\right| \leq \frac{c}{R}\left\{\int_{T_{R}}|\nabla \boldsymbol{u}|^{3 / 2}\right\}^{2 / 3}\left\{\int_{T_{R}} d v\right\}^{1 / 3} \leq\left\{\int_{T_{R}}|\nabla \boldsymbol{u}|^{3 / 2}\right\}^{2 / 3} .
$$

Therefore, letting $R \rightarrow+\infty$ in (36) yields

$$
\int_{\partial \Omega} s(u)=0
$$

and this implies (32).

From

$$
\begin{aligned}
v_{i}[f](x) & =\int_{\mathbb{R}^{3}}\left[\mathcal{u}_{i j}(x-y)-\mathcal{U}(y)-y_{k} \partial_{k} u_{i j}(y)\right] f_{j}(y) d v_{y} \\
& +\mathcal{U}_{i j}(x) \int_{\mathbb{R}^{3}} f_{j}+\partial_{k} \mathcal{U}(x) \int_{\mathbb{R}^{3}} \mathcal{U}_{i j}(y) f_{j}(y) d v_{y}
\end{aligned}
$$

by (33), Lemma 4, Lagrange's theorem and (29)

$$
\nabla \mathcal{V}[f](x)=O\left(r^{-4}\right),
$$

so that $\nabla \mathcal{V}[f] \in L^{1}$. Since $f \in L^{2}\left(\mathbb{R}^{3}\right)$ has compact support and satisfies (33), it belongs to $\mathcal{H}^{1}$ (see [7] p. 92) and by Lemma $2 \mathcal{V}[\boldsymbol{f}] \in \mathcal{H}^{1}$. Hence, it follows that (30) maps $\mathcal{H}^{1}$ into itself and

$$
\left\|\boldsymbol{v}^{\prime}-\boldsymbol{z}^{\prime}\right\|_{\mathcal{H}^{1}} \leq\left\|\mathbf{C}-\mathrm{C}_{0}\right\|_{L^{\infty}\left(\complement S_{R_{0}}\right)}\|\boldsymbol{v}-z\|_{\mathcal{H}^{1}}
$$

Since, by assumptions, $\left\|\mathbf{C}-\mathbf{C}_{0}\right\|_{L^{\infty}\left(C S_{R_{0}}\right)}$ is small, (30) is a contraction and by the above argument its (unique) fixed point must coincide with $v$ so that $\nabla \boldsymbol{u} \in L^{1}(\Omega)$.

We aim at concluding the paper with the following remarks.

(i) It is evident that the hypothesis that $\mathbf{C}$ is regular at infinity can be replaced by the weaker one that $\left|\mathbf{C}-\mathbf{C}_{0}\right|$ is suitably small at a large spatial distance.

(ii) The operator $\mathcal{V}$ maps boundedly the Hardy space $\mathcal{H}^{q}(q \in(0,1])$ into itself [7]. Hence, the argument in the proof of (16) can be used to show that $\nabla v \in \mathcal{H}^{q}, q>3 / 4$. We can then use the Sobolev-Poincaré (see [8] p. 255) to see that $u \in L^{q}(\Omega)$ for $q>1$.

(iii) Relation (16) is a kind of Stokes' paradox in nonhomogeneous elastostatics: if $\boldsymbol{C}$ is regular at infinity, then the system

$$
\begin{array}{rlrl}
\operatorname{div} \boldsymbol{C}[\nabla \boldsymbol{h}] & =\mathbf{0} & \text { in } \Omega, \\
\boldsymbol{h} & =\boldsymbol{\tau} \quad \text { on } \partial \Omega,
\end{array}
$$


with $\tau$ nonzero constant vector, does not admit solutions.

(iv) If $\mathbf{C}$ is constant and strongly elliptic, then $u$ is analytic in $\Omega$ and at large spatial distance admits the representation

$$
\boldsymbol{u}(x)=\boldsymbol{U}(x) \int_{\partial \Omega} \boldsymbol{s}(\boldsymbol{u})+\nabla \mathfrak{U}(x) \int_{\partial \Omega}(\boldsymbol{\xi} \otimes \boldsymbol{s}(\boldsymbol{u})-\mathbf{C}[\hat{\boldsymbol{u}} \otimes \boldsymbol{n}])(\xi)+\boldsymbol{\psi}(x)
$$

with $|x|^{3}|\psi(x)| \leq c$. Therefore, in the homogeneous case, the conclusions of Theorem 1 hold pointwise:

$$
\begin{gathered}
|\boldsymbol{x}|^{2}|\boldsymbol{u}(x)| \leq c \Longleftrightarrow \int_{\partial \Omega} \hat{\boldsymbol{u}} \cdot \boldsymbol{s}(\boldsymbol{h})=\mathbf{0}, \quad \forall \boldsymbol{h} \in \mathfrak{C}_{0} \\
\int_{\partial \Omega} \mathbf{C}[\hat{\boldsymbol{u}} \otimes \boldsymbol{n}]=\mathbf{0}, \quad \int_{\partial \Omega} \hat{\boldsymbol{u}} \cdot \boldsymbol{s}(\boldsymbol{h})=\mathbf{0}, \quad \forall \boldsymbol{h} \in \mathfrak{C} \Rightarrow|\boldsymbol{x}|^{3}|\boldsymbol{u}(x)| \leq c .
\end{gathered}
$$

Funding: This research was funded by FAR 2019 University of Ferrara.

Conflicts of Interest: The author declares no conflict of interest.

\section{References}

1. Gurtin, M.E. The linear theory of elasticity. In Handbuch der Physik; Truesedell, C., Ed.; Springer: Berlin, Germany, 1972; Volume VIa/2.

2. Galdi, G.P. An Introduction to the Mathematical Theory of the Navier—Stokes Equations. Steady-State Problems; Springer: Berlin, Germany, 2011.

3. Fichera, G. Existence theorems in elasticity. In Handbuch der Physik; Truesedell, C., Ed.; Springer: Berlin, Germany, 1972; Volume VIa/2.

4. Russo, R. On Stokes' problem. In Advances in Mathematical Fluid Mechanics; Sequeira, R.R.A., Ed.; Springer: Berlin, Germany, 2010; pp. 473-512.

5. Kondratev, V.A.; Oleinik, O.A. Boundary value problems for a system in elasticity theory in unbounded domains. Korn inequalities. Uspekhi Mat. Nauk 1988, 43, 55-98. (In Russian)

6. John, F. Plane Waves and Spherical Means Applied to Partial Differential Equations; Interscience: New York, NY, USA, 1955.

7. Stein, E. Harmonic Analysis: Real-Variable Methods, Orthogonality, and Oscillatory Integrals; Princeton University Press: Princeton, NJ, USA, 1993.

8. Coifman, R.R.; Lions, P.L.; Meyer, Y.; Semmes, S. Compensated compactness and Hardy spaces. J. Math. Pures Appl. 1993, 72, 247-286.

(C) 2020 by the author. Licensee MDPI, Basel, Switzerland. This article is an open access article distributed under the terms and conditions of the Creative Commons Attribution (CC BY) license (http:/ / creativecommons.org/licenses/by/4.0/). 